\title{
LORSys - Um Sistema de Recomendação de Objetos de Aprendizagem SCORM
}

\author{
Vinicius Hartmann Ferreira \\ André Luís Alice Raabe
}

\begin{abstract}
Resumo
Objetos de aprendizagem são construídos obedecendo um conjunto de especificações que permitem que eles sejam reusáveis em diferentes contextos. LMSs (Learning Management System) como o MOODLE podem assumir o papel de repositório de objetos de aprendizagem. Porém muitas vezes os alunos, usuários destes LMSs, não têm a experiência necessária para encontrar os objetos de aprendizagem que podem contribuir com o seu aprendizado ou não possuem iniciativa para tal. Este artigo apresenta um sistema de recomendação de objetos de aprendizagem no formato SCORM para o MOODLE, que realiza recomendações utilizando as técnicas de filtragem colaborativa e baseada em conteúdo.
\end{abstract}

Abstract

Learning objects are constructed obeying a set of specifications that allow them to be reusable in different contexts. LMSs (Learning Management System) like Moodle could take the role of repository of learning objects. But often the students, users of these LMSs do not have the necessary experience to find learning objects that can contribute to your learning or have no initiative to do so. This article presents a recommendation system of learning objects in SCORM format for Moodle, which performs the technical recommendations using collaborative filtering and content-based.

vinihf@gmail.com; raabe@univali.br

Universidade do Vale do Itajaí, Curso de Ciência da Computação Itajaí, SC

\section{INTRODUÇÃO}

Para suporte aos alunos da modalidade de Ensino a Distância (EaD) a UNIVALI (Universidade do Vale do Itajaí) desenvolveu o Ambiente Sophia, que é uma personalização do MOODLE. Uma das principais tarefas do ambiente é servir de repositório para os objetos de aprendizagem no formato SCORM desenvolvidos para este mesmo público alvo.

A partir do uso continuado deste ambiente durante dois anos, foi possível perceber que muitos alunos não utilizavam os objetos de aprendizagem com a freqüência e intensidade desejadas pelos criadores dos objetos. Acredita-se que em muitos casos o problema ocorre pelo fato dos alunos não possuírem a experiência necessária para encontrar objetos de aprendizagem que contribuam com o seu aprendizado e também por não terem a iniciativa de buscá-los.

Este artigo relata o desenvolvimento de um sistema de recomendação de objetos de aprendizagem no formato SCORM para o Ambiente Sophia/Moodle. Um sistema de recomendação utiliza associações entre itens e também informações de outros usuários para realizar sugestões. O sistema se chama LorSys (Learning Object Recommender System) e as recomendações são geradas através das técnicas de filtragem colaborativa e da filtragem 
baseada em conteúdo. Decidiu-se utilizar estas duas técnicas pelo sucesso obtido em trabalhos similares, conforme visto na seção 5 .

\section{OBJETOS DE APRENDIZAGEM}

Uma forma de apresentar o conteúdo aos alunos, tanto na modalidade presencial ou a distância, é através do uso de recursos digitais, visto os benefícios que o emprego da tecnologia pode produzir. É neste contexto que surgem os objetos de aprendizagem. Segundo Wiley (2001), um objeto de aprendizagem é qualquer entidade digital que pode ser utilizada, reutilizada ou referenciada durante o processo de ensino apoiado pela tecnologia. Um objeto de aprendizagem pode utilizar diversos recursos digitais para ser desenvolvido. Estes recursos incluem vídeos, animações, textos, figuras, músicas, etc.

Ainda segundo Wiley (2001), o conceito de objetos de aprendizagem é desenvolvido baseado no paradigma da ciência da computação de orientação a objeto. Através da orientação a objetos são criados objetos que podem ser reusados em múltiplos contextos. A reusabilidade é uma das idéias fundamentais dos objetos de aprendizagem, permitindo que objetos que são construídos para explicar um conteúdo de uma determinada disciplina possa ser utilizado para complementar outro assunto de outra disciplina. Isto se dá quando observa-se que quanto menor o objeto (mais especializado no assunto e não no contexto em que será empregado) maior é a probabilidade de ele ser utilizado em outro contexto. Outra característica dos objetos de aprendizagem é o fato de ser disponibilizado através da internet, o que permite que um grande número de pessoas tenha acesso a ele.

Para que os objetos de aprendizagem possam ser identificados e classificados são utilizados metadados (dados sobre dados). Os metadados podem guardar informações sobre os autores, o conteúdo abordado, a descrição do objeto, etc. Segundo Hansen e Pinto (2003), o ensino a distância pode se beneficiar da utilização de metadados, pois a descrição dos recursos digitais utilizados permite que sejam desenvolvidas aplicações interoperáveis e o compartilhamento de recursos educacionais entre diferentes sistemas é propiciado através da descrição formal de suas interfaces.

A descrição dos objetos de aprendizagem através de metadados não seria de total proveito se esta não seguisse um padrão. Existem diversos padrões de metadados, entre eles Dublin Core, CanCore e LOM (Learning Object Metadata), ambos armazenados em XML. Segundo Hansen e Pinto (2003) o LOM se caracteriza por permitir a definição de blocos independentes, compartilhamento e troca de material educacional.

\section{SCORM}

A fim de modernizar e padronizar a disponibilização de material educacional o departamento de defesa dos Estados Unidos da América criou a iniciativa ADL, que veio a desenvolver o SCORM (Sharable Content Object Reference Model) (ADLNET, 2008). O SCORM é uma coleção de padrões e especificações que define como o Objeto de Aprendizagem deve ser organizado e "empacotado” para ser aberto por um LMS, gerando assim acessibilidade, interoperabilidade, reusabilidade e durabilidade ao conteúdo produzido.

Definindo-se estas vantagens da utilização do padrão SCORM como:

- Acessibilidade: A habilidade de localizar e acessar componentes em múltiplos ambientes e entregá-los para outros ambientes.

- Interoperabilidade: A habilidade de utilizar um componente desenvolvido para um ambiente em outro. 
- Durabilidade: A capacidade de resistir a evolução tecnológica e/ou sofrer mudanças sem custos com re-planejamento, reconfiguração ou recodificação.

- Reusabilidade: A flexibilidade em incorporar componentes instrucionais em múltiplos contextos e aplicações.

Segundo Bohl et al (2002), o objetivo fundamental do padrão SCORM é possibilitar facilmente a portabilidade de material educacional entre um LMS e outro, fazendo valer também a característica de reusabilidade dos objetos de aprendizagem.

\section{SISTEMAS DE RECOMENDAÇÃO}

Segundo Schafer (1999) o aumento da possibilidade de disponibilização de conteúdo (produtos ou informação) através de sistemas web, faz com que os sistemas web apresentem mais opções de escolha para o usuário antes de ele estar apto a encontrar a opção que vai ao encontro de sua necessidade. Uma das soluções para resolver este problema de sobrecarga de informação é a utilização de sistemas de recomendação.

Segundo Burke (2002) um sistema de recomendação pode ser qualquer sistema que produza recomendações individuais ao usuário ou que tenha o efeito de guiar o usuário através de recomendações personalizadas a itens que sejam de seu interesse ou que o sejam úteis diante de uma vasta gama de opções. Um sistema de recomendação pode também identificar similaridade entre o comportamento dos usuários e recomendar itens, como objetos de aprendizagem, que já foram recomendados para outros usuários. Existem diversas técnicas utilizadas para realizar recomendações. A seguir serão apresentadas as técnicas de filtragem colaborativa e filtragem baseada em conteúdo.

\subsection{Filtragem Colaborativa}

Segundo Burke (2002), a filtragem colaborativa é a técnica mais conhecida, mais implementada e mais madura de todas. Ela reúne as notas ou recomendações atribuídas pelos usuários aos itens, identifica as similaridades entre os usuários baseado em suas avaliações e gera novas recomendações com base em comparações entre os usuários.

Segundo Lichtnow et al (2006), define-se que usuários com perfil similar são os usuários que avaliaram os mesmo itens de forma similar. O sistema armazena as notas que o usuário atribui para cada item visitado, e permite que um determinado grupo formado pelos interesses em comum possa visualizar a avaliação que este usuário deu para cada item. Segundo Schafer (1999), esta técnica de recomendação pode ser chamada de recomendação de "pessoa para pessoa".

A filtragem colaborativa possui as vantagens de que o sistema não analisa o conteúdo dos itens avaliados, evitando uma complexidade maior na sua implementação e facilitando a recomendação de itens como músicas e jogo onde a variação nos gostos do usuário influencia na variação das suas preferências, e também pode realizar recomendações inesperadas ao usuário. Porém para que as recomendações sejam relevantes é necessário que haja uma grande quantia de usuários no sistema e usuários que possuam interesses não muito comuns receberão recomendações pobres.

\subsection{Filtragem Baseada em Conteúdo}

Segundo Lichtnow et al (2006) a filtragem por conteúdo parte do princípio de que os usuários tendem a se interessar por itens que se interessaram no passado, tornando-se 
necessária a identificação de associação entre os itens. Diferente da filtragem colaborativa, esta técnica analisa o conteúdo dos itens. A recomendação realizada por esta técnica ocorre através da associação entre a similaridade entre o conteúdo dos itens e a análise dos interesses do usuário ou do conteúdo dos itens já avaliados pelo usuário.

Segundo Schafer (1999), esta técnica pode ser chamada de recomendação de "item para item”, pois baseia-se no conteúdo dos itens. Segundo Shardanand e Maes (1995), está técnica não realiza a avaliação qualitativa dos itens que são recomendados, pois basta que haja similaridade entre os atributos que são comparados para que o item seja recomendado para o usuário.

A técnica de filtragem baseada em conteúdo apresenta fragilidade na recomendação de itens com pouca descrição, pois é baseado nesta descrição dos itens que o sistema realiza a recomendação ao usuário. A descrição dos itens deve ser bem elaborada, pois até mesmo a utilização de sinônimos pode gerar recomendações erradas.

\section{Trabalhos Similares}

A Tabela 1 apresenta trabalhos similares ao que este trabalho desenvolveu.

\begin{tabular}{|c|c|c|c|c|}
\hline Título & Escopo & $\begin{array}{l}\text { Técnicas } \\
\text { utilizadas }\end{array}$ & $\begin{array}{l}\text { Parâmetros para } \\
\text { recomendação }\end{array}$ & Avaliação \\
\hline $\begin{array}{c}\text { S. R. para } \\
\text { Bibliotecas Digitais } \\
\text { (Lopes, 2006) }\end{array}$ & $\begin{array}{l}\text { Recomenda artigos } \\
\text { científicos. }\end{array}$ & $\begin{array}{l}\text { Filtragem } \\
\text { baseada em } \\
\text { conteúdo. }\end{array}$ & $\begin{array}{l}\text { 1.I Informações do } \\
\text { currículo lattes; e } 2 \text {. } \\
\text { Informações do item. }\end{array}$ & $\begin{array}{l}\text { Avaliação dos } \\
\text { itens } \\
\text { recomendados. }\end{array}$ \\
\hline $\begin{array}{c}\text { SisReCol } \\
\text { (Lichtow, 2006) }\end{array}$ & $\begin{array}{l}\text { Recomenda sites } \\
\text { documentos e } \\
\text { autoridades no } \\
\text { assunto. }\end{array}$ & $\begin{array}{l}\text { Filtragem } \\
\text { colaborativa e } \\
\text { baseada em } \\
\text { conteúdo. }\end{array}$ & $\begin{array}{c}\text { Mensagens trocadas } \\
\text { entre os usuários do } \\
\text { chat e interação com } \\
\text { ambiente. }\end{array}$ & $\begin{array}{c}\text { Precisão e } \\
\text { abrangência. }\end{array}$ \\
\hline $\begin{array}{c}\text { Fab } \\
\text { (Balabanovic, } \\
\text { 1997) }\end{array}$ & $\begin{array}{l}\text { Recomenda } \\
\text { páginas Web para } \\
\text { usuários. }\end{array}$ & $\begin{array}{l}\text { Filtragem híbrida } \\
\text { (colaborativa e } \\
\text { baseada em } \\
\text { conteúdo). }\end{array}$ & $\begin{array}{l}\text { Tópico de interesse do } \\
\text { usuário. }\end{array}$ & $\begin{array}{c}\text { Semelhança } \\
\text { entre o perfil } \\
\text { dos usuários e } \\
\text { o dos agentes } \\
\text { de seleção. }\end{array}$ \\
\hline $\begin{array}{c}\text { Mobile } \\
\text { Recommender } \\
\text { System } \\
\text { (Ricci,2007) }\end{array}$ & $\begin{array}{l}\text { Recomenda } \\
\text { produtos para } \\
\text { viagem (hotel, } \\
\text { restaurante, ...). }\end{array}$ & $\begin{array}{l}\text { Filtragem } \\
\text { baseada em } \\
\text { conteúdo. }\end{array}$ & $\begin{array}{l}\text { Opiniões do usuário } \\
\text { sobre um restaurante. } \\
\text { Estas opiniões variam } \\
\text { entre wish e must. }\end{array}$ & Questionário. \\
\hline $\begin{array}{c}\text { Dual Information } \\
\text { Sources } \\
\text { Collaborative } \\
\text { Filtering } \\
\text { (Cho, 2007) }\end{array}$ & $\begin{array}{l}\text { Recomenda Videos } \\
\text { sob demanda } \\
\text { (VoD) no contexto } \\
\text { de elearning. }\end{array}$ & $\begin{array}{c}\text { Filtragem } \\
\text { colaborativa } \\
\text { baseada em duas } \\
\text { fontes. }\end{array}$ & $\begin{array}{l}\text { Comportamento do } \\
\text { usuário (clique, } \\
\text { visualização e } \\
\text { pagamento). }\end{array}$ & Precisão. \\
\hline $\begin{array}{l}\text { S.R. para análise } \\
\text { de produtos pelos } \\
\text { usuários } \\
\text { (Aciar, 2007) }\end{array}$ & $\begin{array}{c}\text { Recomenda } \\
\text { produtos na } \\
\text { internet (câmeras } \\
\text { digitais, etc...) }\end{array}$ & $\begin{array}{l}\text { Text-mining., } \\
\text { Ontologias, Grau } \\
\text { de experiência. }\end{array}$ & $\begin{array}{c}\text { Comentários do usuário } \\
\text { e seu grau de } \\
\text { experiência. }\end{array}$ & $\begin{array}{l}\text { Precisão e } \\
\text { Revocação. }\end{array}$ \\
\hline $\begin{array}{c}\text { RecS-DL } \\
\text { (Pedronette, 2008) }\end{array}$ & $\begin{array}{c}\text { Recomenda } \\
\text { materiais } \\
\text { disponibilizados em } \\
\text { bibliotecas digitais. }\end{array}$ & $\begin{array}{l}\text { Novas técnicas } \\
\text { podem ser } \\
\text { implementadas } \\
\text { como plugins. }\end{array}$ & Itens, Usuários, Ratings. & Questionário. \\
\hline $\begin{array}{c}\text { LorSys } \\
\text { (Omitido, 2009) }\end{array}$ & $\begin{array}{l}\text { Recomenda objetos } \\
\text { de aprendizagem }\end{array}$ & $\begin{array}{l}\text { Filtragem } \\
\text { colaborativa e } \\
\text { baseada em } \\
\text { conteúdo. }\end{array}$ & $\begin{array}{l}\text { Metadados dos objetos } \\
\text { de aprendizagem e } \\
\text { informações sobre a } \\
\text { utilização pelos alunos. }\end{array}$ & $\begin{array}{l}\text { Qualitativas e } \\
\text { quantitativas. }\end{array}$ \\
\hline
\end{tabular}

Tabela 1. Tabela comparativa de trabalhos similares 
Através da Tabela 1 é possível observar a diversidade de sistemas de recomendação desenvolvidos atualmente. As técnicas de recomendação mais utilizadas são a técnica de filtragem baseada em conteúdo e a técnica de filtragem colaborativa.

Todos os sistemas apresentados que utilizam a filtragem colaborativa disponibilizam ao usuário um método simples para o usuário avaliar itens. Através desta avaliação o sistema pode atualizar o perfil do usuário com os assuntos de seu interesse e então realizar a recomendação ou realizar a recomendação baseando-se na avaliação em si.

Quando os itens alvos da recomendação possuem metadados que os descrevem, como é o caso dos objetos de aprendizagem, é mais viável a utilização da filtragem baseada em conteúdo. Através dos metadados é possível facilmente classificar e identificar similaridade entre os interesses do usuário e o assunto tratado pelo item.

Os parâmetros utilizados pelos sistemas para gerar recomendações não se diferenciam muito um do outro. Em sua maioria utilizam a avaliação que um usuário deu a um item (filtragem colaborativa) ou o conteúdo do item e o perfil do usuário (filtragem baseada em conteúdo). Porém a forma como esses parâmetros são obtidos pelo sistema podem variar entre um simples sistema de avaliação de itens ou técnicas de text-mining que extraem informações de comentários feitos pelo usuário.

Para a avaliação dos resultados obtidos o uso de questionários trouxe evidências mais confiáveis, pois através do questionário é possível receber a opinião dos usuários sobre as recomendações que o sistema gerou, bem como se o sistema é intuitivo ou se as recomendações são apresentadas no momento oportuno e da forma adequada. Embora existam algoritmos que possam avaliar a precisão e a revocação (recall) de uma recomendação, não se pode comparar a avaliação dada pelo "cliente" do sistema, visto que muitas vezes uma recomendação pode ser precisa no aspecto técnico, mas não ir ao encontro da necessidade do usuário.

No contexto educacional podem ser citados o Sistema de Recomendação para Bibliotecas Digitais, o SisReCol e o RecS-DL, que foram desenvolvidos com o objetivo de recomendar material instrucional como artigos encontrados em bibliotecas digitais, ou autoridades em determinado assunto. O DISCORS e o RACOFI podem ser citados também pela abrangência de suas recomendações, que além de outros itens podem recomendar também material instrucional.

Através do estudo dos trabalhos similares, foi definido que o sistema de recomendação desenvolvido utilizaria as técnicas de filtragem colaborativa e de filtragem baseada em conteúdo. Pois o Ambiente Sophia registra a navegação do usuário, permitindo assim identificar similaridade entre usuários e os objetos de aprendizagem possuem metadados que os descrevem, o que permite identificar a similaridade entre seus conteúdos.

\section{LORSys}

O LORSys (Learning Object Recommender System) é um sistema de recomendação de objetos de aprendizagem no padrão SCORM para o MOODLE que utiliza as técnicas de filtragem colaborativa e filtragem baseada em conteúdo para gerar recomendações. Estas foram as técnicas escolhidas porque o MOODLE e os objetos de aprendizagem no padrão SCORM disponibilizam informações suficientes para utilizá-las.

O MOODLE armazena informações sobre a utilização dos objetos de aprendizagem por parte dos usuários, proporcionando as informações necessárias para a utilização da filtragem colaborativa. Os objetos de aprendizagem no padrão SCORM possuem metadados que o descrevem, proporcionando informações suficientes para a utilização da técnica baseada em conteúdo. 
A descrição do LORSys apresentada nesta seção está organizada da seguinte forma: (i) aquisição de informação; (ii) recomendação; (iii) visualização das recomendações; e (iv) avaliação.

\subsection{Aquisição de Informação}

O módulo de aquisição de informação é responsável por coletar e armazenar informações encontradas nos metadados dos objetos de aprendizagem SCORM do Ambiente Sophia e também por armazenar informações sobre a utilização destes objetos de aprendizagem por parte dos usuários.

Quando um usuário utilizar um objeto de aprendizagem por mais de 20 segundos, o módulo de aquisição armazenará em uma tabela do banco de dados denominada tabela de preferência o identificador deste objeto.

\subsection{Recomendação}

A recomendação é feita baseada nas informações contidas na tabela de preferência do estudante e utiliz duas técnicas: a filtragem baseada em conteúdo e a filtragem colaborativa.

\subsubsection{Recomendação por Filtragem Baseada em Conteúdo}

Serão recomendados aos usuários objetos que possuem relação com o conteúdo do último objeto acessado pelo usuário. Para identificar as relações entre os objetos o sistema provê uma interface ao professor da disciplina, como pode ser visto na figura abaixo, em que ele deverá definir o grau de relacionamento entre os objetos de aprendizagem que foram inseridos no MOODLE, classificando-os em: (i) Pré-Requisito; (ii) Complemento; (iii) Similar; e (iv) Sem relação. A interface exibe na primeira parte informações sobre o objeto de aprendizagem alvo das recomendações e abaixo os demais objetos de aprendizagem e o grau de relação entre eles e o objeto alvo.

\begin{tabular}{|c|c|c|c|}
\hline Titulo: & \multicolumn{3}{|l|}{ Árvore Binária de Busca } \\
\hline Assunto: & \multicolumn{3}{|l|}{ Estrutura de Dados, Árvore Binária de Busca, Aplicativo } \\
\hline Descrição: & \multicolumn{3}{|c|}{$\begin{array}{l}\text { Permite ao acadêmico visualizar e interagir com a estrutura de dados de uma } \\
\text { Árvore Binária de Busca. }\end{array}$} \\
\hline Autor: & \multicolumn{3}{|c|}{ Laboratório de Soluções em Software Organizador: UNIVALI } \\
\hline \multicolumn{4}{|c|}{$\begin{array}{l}\text { Contribuição: Vinicius H. Ferreira (Produtor do Objeto), Elieser Ademir de Jesus } \\
\text { (Desenvolvedor do Aplicativo), Rudimar Luís Scaranto Dazzi (Desenvolvedor } \\
\text { do Aplicativo), Fabiane Vavassori (Produtora do Objeto). }\end{array}$} \\
\hline Licença: & \multicolumn{3}{|c|}{$\begin{array}{l}\text { (BY-NC-ND)Esta obra está licenciada sob uma Licença Creative Commons } \\
\text { (http://creativecommons.org/licenses/by-nc-nd/3.0/deed.pt) }\end{array}$} \\
\hline \multicolumn{2}{|l|}{ Título } & Relação & \\
\hline \multicolumn{2}{|c|}{ Utilizando estrutura de dados Lista: desafio Controle de Lista de Espera Companhia Aérea } & Sem relação & $\checkmark$ \\
\hline \multicolumn{2}{|c|}{ Utilizando estrutura de dados Pilha: desafio do Armazém } & Pré-Requisito & $\boldsymbol{\nabla}$ \\
\hline \multicolumn{2}{|c|}{ Preencha as lacunas } & Complemento & 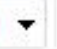 \\
\hline & Relacionar & & \\
\hline
\end{tabular}

Relacionamento de objetos de aprendizagem 


\subsubsection{Recomendação por Filtragem Colaborativa}

A filtragem colaborativa é realizada de duas formas, (i) quando um usuário acessar o curso o sistema identificará usuários que acessaram os mesmos objetos que ele, feito isso o sistema irá recomendar o último objeto de aprendizagem acessado por estes usuários; e (ii) através das informações de utilização dos objetos de aprendizagem pelos usuários armazenadas na tabela de preferência, o sistema recomendará o objeto de aprendizagem que foi mais acessado. Este processo se caracteriza como uma Filtragem Colaborativa pelo fato de o sistema gerar as recomendações baseando-se na colaboração entre a turma na utilização dos objetos de aprendizagem.

Quando um objeto de aprendizagem for recomendado ao mesmo tempo pelas duas técnicas de recomendação ele será classificado e recomendado como dica quente.

\subsection{Visualização das Recomendações}

A visualização da recomendação é feita através de um bloco (recurso do MOODLE), adicionado no curso. Na figura abaixo é possível visualizar como são apresentadas as recomendações aos usuários.

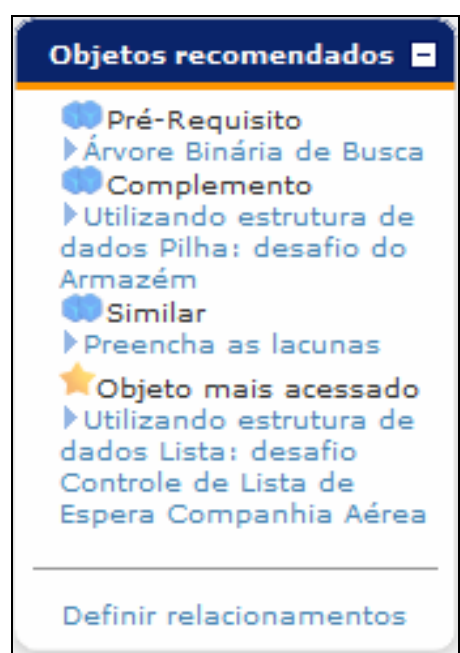

Bloco LORSys

\subsection{Avaliação}

A fim de avaliar o LorSys, foi disponibilizado a um pequeno grupo de usuários o acesso a um curso que possuirá objetos de aprendizagem na área de Ciência da Computação. Foi solicitado aos usuários que interagissem diariamente com os objetos de aprendizagem disponibilizados no ambiente durante duas semanas.

Avaliação foi realizada de duas formas: quantitativa e qualitativa. Na avaliação quantitativa foram analisadas quantas recomendações foram acatadas pelos usuários, ou seja, quantos cliques foram dados sobre links recomendados pelo sistema. Esta informação permitiu realizar a comparação entre os tipos diferentes de filtragem usados e sua eficiência e também analisar a evolução do sistema de acordo com o crescimento de informações armazenadas. A avaliação qualitativa foi realizada perguntando aos usuários como eles avaliam ao final do período do experimento as recomendações feitas pela ferramenta. 
Através da avaliação quantitativa, foi possível identificar que as recomendações geradas através da filtragem baseada em conteúdo foram mais aceitos. Esta situação pode ter ocorrido pelo fato de estas recomendações serem exibidas no topo do bloco, pelo fato de haver sempre recomendação nesta categoria, já que é o professor que estabelece as relações e não o sistema, ou pelo fato de haver relação entre os conteúdos.

Na avaliação qualitativa os usuários aprovaram o funcionamento do LorSys, apenas sugerindo uma melhor forma de relacionar os objetos e salientando o fato de 0 aproveitamento do bloco ser melhor em disciplinas com vários objetos de aprendizagem.

\section{CONSIDERAÇÕES FINAIS}

Acredita-se que as técnicas de recomendação implementadas no LorSys (filtragem colaborativa e baseada em conteúdo) atendem de forma eficiente o público alvo das recomendações. Através da técnica de filtragem baseada em conteúdo os usuários recebem a recomendação de objetos de aprendizagem que complementem seu aprendizado de diversas formas (pré-requisito, similar, e complemento). E através da técnica de filtragem colaborativa os usuários recebem recomendações de objetos de aprendizagem acessados por usuários que acessaram o mesmo objeto que ele.

As duas técnicas de recomendação se complementam, pois enquanto uma se aperfeiçoa com a quantidade de usuários interagindo (filtragem colaborativa) a outra se aperfeiçoa com a interação individual do usuário (filtragem baseada em conteúdo).

Também entende-se que a aplicabilidade do bloco LorSys justifica-se em um contexto em que vários usuários de uma disciplina possuem acesso a uma vasta quantidade de objetos de aprendizagem e não sabem quais objetos de aprendizagem lhe acrescentarão conhecimento ou não tem iniciativa em fazê-lo. Com seu desenvolvimento direcionado ao Ambiente Sophia este contexto tende a ser cada vez mais freqüente, já que o Ambiente Sophia tem por objetivo futuro atender a todos os cursos de graduação e ao colégio de aplicação da UNIVALI.

\section{REFERÊNCIAS}

ACIAR, Silvana et al. Informed recommender: basing recommendations on consumer product reviews. IEEE Intelligent Systems, v. 26, n. 3, maio e jun. 2007.

ADLNET. Advanced distributed learning - SCORM. Disponível em: < http://www.adlnet.gov/scorm/index.aspx >. Acesso em 18 mar. 2009.

BALABANOVIC, Marko; SHOHAM, Yoav. FAB: a content-based, collaborative recommendation. Communications of the ACM, v.40, n.3, p. 66-72, march. 1997.

BOHL, Oliver et al. The sharable content reference model (SCORM) - a critical review. In: INTERNATIONAL CONFERENCE ON COMPUTERS IN EDUCATION. Auckland, New Zealand, 2002, Proceedings... IEEE Computer Society, 2002, ISBN 07695-1509-6, vol. 2

BURKE, Robin. Hybrid recommender systems: survey and experiments. User Modeling and User-Adapted Interaction, Hingham, MA, EUA, v. 12, n. 4, p.331-370, 2002.

CHO, Jinhyung et al. Collaborative filtering using dual information sources. In: IEEE Intelligent Systems, v. 26, n. 3, maio e jun. 2007.

HANSEN, Roseli P.; PINTO, Sérgio Crespo S. C. Construindo ambientes de educação baseada na web através de web services educacionais. In: SIMPÓSIO BRASILEIRO DE INFORMÁTICA NA EDUCAÇÃO, 14. , nov. 2003, Rio de Janeiro/RJ, UFRJ.

LICHTNOW, Daniel et al. O uso de técnicas de recomendação para apoio à aprendizagem 
colaborativa. Revista Brasileira de Informática na Educação. vol. 14, n.3, set. 2006.

LOPES, Giseli Rabello et al. Sistema de recomendação para bibliotecas digitais sob a perspectiva da web semântica. WORKSHOP DE BIBLIOTECAS DIGITAIS, 2006.

PEDRONETTE, D.; TORRES, R. Uma plataforma de serviços de recomendação para bibliotecas digitais. SIMPÓSIO BRASILEIRO DE BANCO DE DADOS, 2008.

RICCI, F.; NGUYEN, Quang Nhat. Acquiring and revising preferences in a critique-based mobile recommender system. IEEE Intelligent Systems, v.26, n.3, 2007.

SCHAFER, J. Ben et al. Recommender systems in e-commerce. In: ACM CONFERENCE ON ELETRONIC COMMERCE, 1., 1999. Denver, Colorado, Estados Unidos da América. Proceedings...Denver: ACM, 1999. p. 158-166.

SHARDANAND, Upendra; MAES, Pattie. Social information filtering: algorithms for automating "word of mouth". In: CONFERENCE ON HUMAN FACTORS IN COMPUTING SYSTEMS, 1995, Denver, Colorado, Estados Unidos da América. Proceedings...Denver: ACM, 1995. p. 210-217.

WILEY, David A. Connecting learning objects to instructional design theory: a definition, a metaphor, and a taxonomy. 2001. Disponível em: <http://reusability.org/read/chapters/wiley.doc>. Acesso em: 6 mar. 2009. 